\title{
Mitochondrial Fuel Flexibility Assessment in a Tumor-Relevant Model
}

David L. Hoffman, Cayman Chemical Company I 1180 East Ellsworth Road, Ann Arbor, Michigan 48108 USA I www.caymanchem.com

HIGHLIGHTS

- Examines metabolic flexibility of cancer cells and human MDSCs in a simulated tumor microenvironment

- Demonstrates the power of combining Seahorse XFe96 technology with manipulation of the major mitochondrial fuel pathways

\section{INTRODUCTION}

The tumor microenvironment (TME) consists of low $\left[\mathrm{O}_{2}\right]$, low pH, and high concentrations of fatty acids. The high amounts of fatty acids are produced by cancer cells as a metabolic by-product. Myeloid-derived suppressor cells (MDSCs) thrive on these high fatty acid concentrations via fatty acid oxidation (FAO). Under these conditions, they support tumor growth by suppressing $T$ cells that would normally attack the tumor.

Although not their primary mechanism, certain chemotherapeutic agents inhibit FAO, sensitizing cells within the TME to death by starvation. ${ }^{1,2}$ Though often difficult to simulate, ${ }^{3}$ it is useful to reproduce the unique environment of the TME in vitro to give a better understanding of how cells behave in this environment. We demonstrate the ability to replicate the atmospheric conditions of the TME using a Seahorse XFe96 to create a tumor-relevant model. We also use a combination of compounds to inhibit transporters of the main mitochondrial fuel pathways (Figure 1). These compounds are used to examine the flexibility (capacity and dependency) of cancer cells' and primary human MDSCs' reliance on pyruvate oxidation versus glutamine metabolism versus FAO by performing a Mitochondrial Fuel Flex Test.

Figure 1. Three pathway inhibitors demonstrate the flexibility of cells to use glucose, glutamine, and fatty acids as mitochondrial fuel sources. MPC=mitochondrial pyruvate carrier; $\mathrm{CPT}=$ carnitine palmitoyltransferase; GLS=glutaminase.

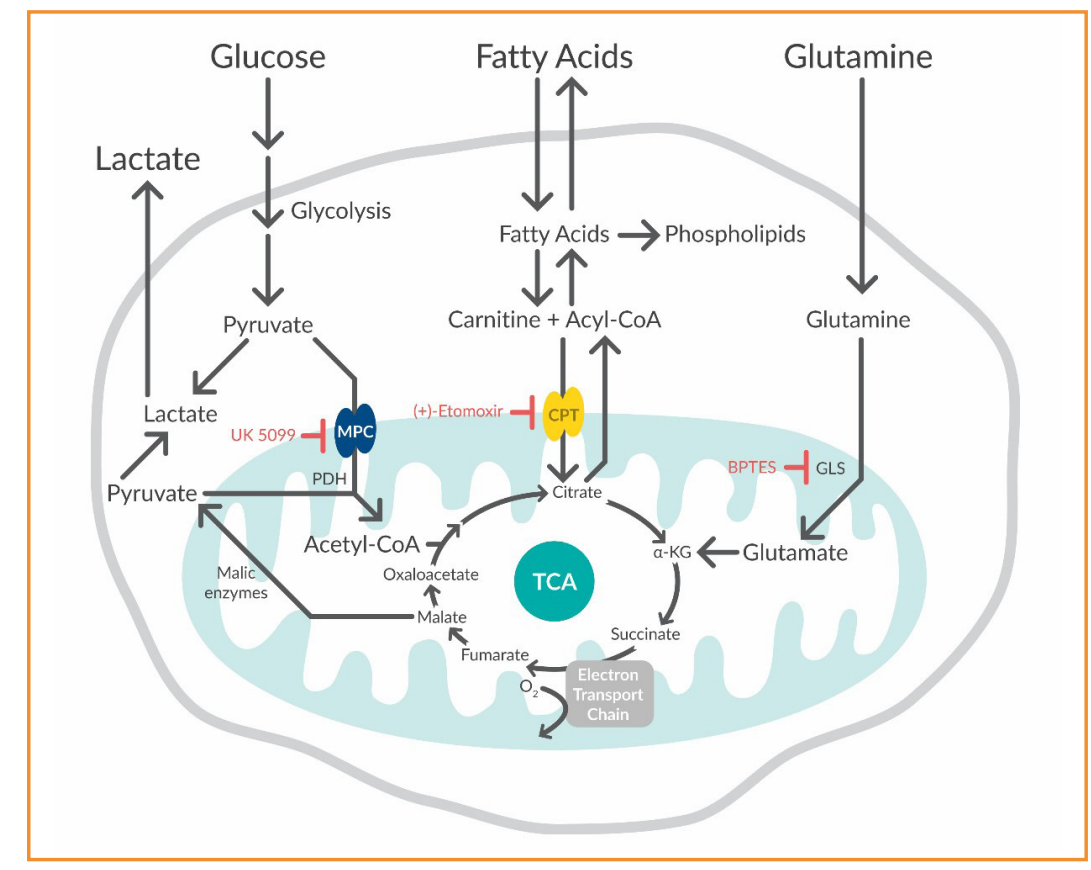

\section{METHODS}



Figure 2. Method workflow for the Mitochondrial Fuel Flex Test.

- HCT116 human colon carcinoma cell culture - XFe96 plates were treated with the chemotherapeutic agent SN-38 or vehicle and maintained at either ambient $\left(21 \% \mathrm{O}_{2} / 5 \% \mathrm{CO}_{2}\right)$ or low $\left(1 \% \mathrm{O}_{2} / 5 \% \mathrm{CO}_{2}\right)\left[\mathrm{O}_{2}\right]$ (Figure 2).

- MDSC isolation - MDSCs were differentiated from monocytes isolated from whole blood. After 4 days, cells were placed at $1 \% \mathrm{O}_{2} / 5 \% \mathrm{CO}_{2}$ or maintained at $21 \% \mathrm{O}_{2} / 5 \% \mathrm{CO}_{2}$ for the remainder of the differentiation period ( 6 days total) before being analyzed.

- Mitochondrial Fuel Flex Test - Capacity and dependency for FAO, glutamine, and pyruvate were determined through the injection of (+)-etomoxir $(2 \mu \mathrm{M})$ to inhibit CPT1 (FAO), UK $5099(4 \mu \mathrm{M})$ to inhibit MPC (mitochondrial pyruvate oxidation), and BPTES (3 $\mu \mathrm{M})$ to inhibit GLS (glutamine oxidation). Oxygen consumption rate (OCR) was measured using a Seahorse XFe96. OCR values at points of equilibrium were used to calculate a percentage of substrate utilization for pyruvate oxidation, glutamine metabolism, and FAO after exposure to various combinations of inhibitor sets. 


\section{RESULTS}

When HCT116 cells were treated with SN-38 acutely or for 12 hours, substrate capacity for pyruvate oxidation, glutamine metabolism, and FAO did not change significantly (data not shown). SN-38 treatment did, however, decrease HCT116 cell dependence on FAO at $21 \% \mathrm{O}_{2}$ (Figure $3 \mathrm{~A}$ and $\mathrm{C}$ ), suggesting that under ambient oxygen concentrations HCT116 cells are more dependent on FAO. At $1 \% \mathrm{O}_{2}$, exposure to SN-38 increased HCT116 cell dependence on pyruvate (Figure 3B and D). This was expected since upregulated glycolysis increases pyruvate availability. Thus, dependency on FAO can be diminished by $\mathrm{SN}-38$ treatment or by exposure to low $\left[\mathrm{O}_{2}\right]$.

In contrast to this, MDSCs cultured at $21 \% \mathrm{O}_{2}$ appear to have increased dependency on glutamine (Figure 4). At $1 \% \mathrm{O}_{2}$, however, a shift in substrate dependency in favor of FAO was observed. This switch in substrate dependencies at $21 \% \mathrm{O}_{2}$ compared to $1 \% \mathrm{O}_{2}$ suggests that lower $\left[\mathrm{O}_{2}\right.$ ] alters the metabolic phenotype of MDSCs.

\section{CONCLUSIONS}

Culture conditions, particularly oxygen concentration, can have a significant impact on metabolic substrate utilization. The ability to assess mitochondrial fuel flexibility in a controlled environment using a combination of inhibitors that pinpoint specific metabolic pathways presents the opportunity to observe cells under conditions that are a better representation of a native environment. In this case, if dependency on mitochondrial fuel sources was not considered when assessing the effects of low $\left[\mathrm{O}_{2}\right]$ on mitochondrial function in the presence of chemotherapeutics, nuanced alterations in metabolic phenotype would not have been readily detected.

With a critical understanding of how substrate utilization and metabolic activity are reprogrammed, new strategies can be developed to modulate the enzymes and transporters driving mitochondrial oxidation pathways. Knowing the factors that affect the dysregulation of glucose, lipid, and/or amino acid homeostasis during diseases such as diabetes, obesity, fatty liver diseases, mitochondrial disorders, cardiac failure, neurodegeneration, and cancer will improve the discovery of targeted therapeutics.

Experiments were conducted in Cayman's Cellular Metabolism Contract Services Lab. For more information on this application and other services offered by Cayman, please contact contractresearch@caymanchem.com or visit www.caymanchem.com/services.

\section{REFERENCES}

1. Schumacher, J.D. and Guo, G.L. Mechanistic review of drug-induced steatohepatitis. Toxicol. Appl. Pharmacol. 289(1), 40-47 (2015).

2. Begriche, K., Massart, J., Robin, M.A., et al. Drug-induced toxicity on mitochondria and lipid metabolism: Mechanistic diversity and deleterious consequences for the liver. J. Hepatol. 54(4), 773-794 (2011).

3. Vander Heiden, M.G. Exploiting tumor metabolism: Challenges for clinical translation. J. Clin. Invest. 123(9), 3648-3651 (2013).

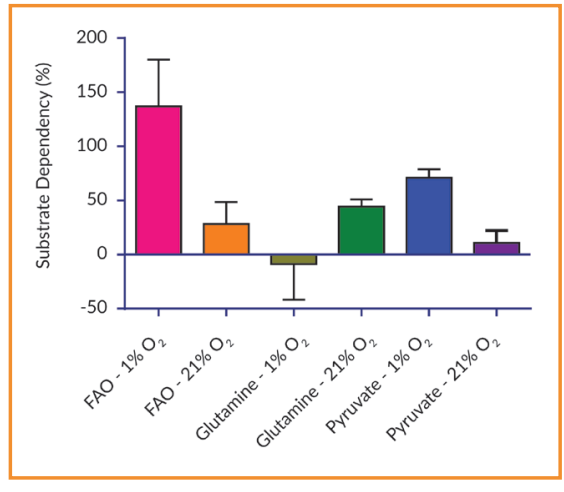

Figure 4. MDSCs differentiated at $21 \%$ and $1 \% \mathrm{O}_{2}$ were subjected to a mitochondrial fuel flex test. Data are presented as means \pm standard error.

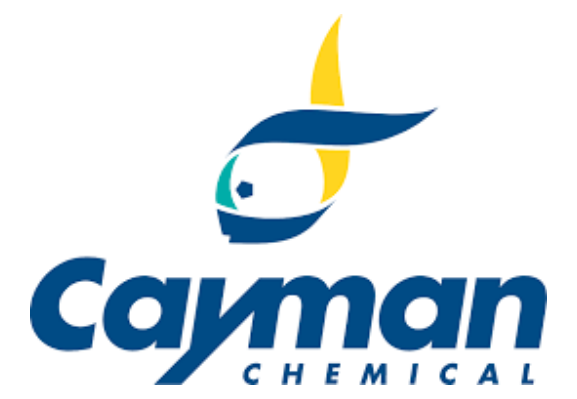

